\title{
The Knockout of Secretin in Cerebellar Purkinje Cells Impairs Mouse Motor Coordination and Motor Learning
}

\author{
Li Zhang', Sookja Kim Chung ${ }^{2}$ and Billy Kwok Chong Chow*, \\ 'School of Biological Sciences, University of Hong Kong, Hong Kong SAR, China; ²Department of Anatomy, University of Hong Kong, Hong Kong \\ SAR, China
}

\begin{abstract}
Secretin (SCT) was first considered to be a gut hormone regulating gastrointestinal functions when discovered. Recently, however, central actions of SCT have drawn intense research interest and are supported by the broad distribution of SCT in Specific neuronal populations and by in vivo physiological studies regarding its role in water homeostasis and food intake. The direct action of SCT on a central neuron was first discovered in cerebellar Purkinje cells in which SCT from cerebellar Purkinje cells was found to potentiate GABAergic inhibitory transmission from presynaptic basket cells. Because Purkinje neurons have a major role in motor coordination and learning functions, we hypothesize a behavioral modulatory function for SCT. In this study, we successfully generated a mouse model in which the SCT gene was deleted specifically in Purkinje cells. This mouse line was tested together with SCT knockout and SCT receptor knockout mice in a full battery of behavioral tasks. We found that the knockout of SCT in Purkinje neurons did not affect general motor ability or the anxiety level in open field tests. However, knockout mice did exhibit impairments in neuromuscular strength, motor coordination, and motor learning abilities, as shown by wire hanging, vertical climbing, and rotarod tests. In addition, SCT knockout in Purkinje cells possibly led to the delayed development of motor neurons, as supported by the later occurrence of key neural reflexes. In summary, our data suggest a role in motor coordination and motor learning for SCT expressed in cerebellar Purkinje cells. Neuropsychopharmacology (2014) 39, I460-1468; doi:I0.1038/npp.2013.344; published online I2 February 20I4
\end{abstract}

Keywords: purkinje specific secretin knockout; motor coordination and learning; motor reflexes; neural development

\section{INTRODUCTION}

Motor coordination and motor learning are essential for animal survival and involve the complex coordination of various functions, including sensory inputs, integration in the central nervous system, and outputs to peripheral skeletal muscles. Numerous studies have established the importance of the cerebellum in motor coordination and learning (Dow and Moruzzi, 1958; Ito, 2002; Swinny et al, 2005; Thach et al, 1992). Within the cerebellar cortex, Purkinje cells function as the sole integrating center: they receive and integrate excitatory inputs from parallel fibers and mossy fibers as well as inhibitory signals from basket cells and stellate cells, resulting in the sole inhibitory output to the deep cerebellar nuclei, which in turn project axons to the vestibular nucleus of the brainstem (Ito, 1984; Lamont and Weber, 2012). The pivotal roles of Purkinje cells in motor coordination and learning functions have been established through various transgenic animal models (GrusserCornehls and Baurle, 2001; Lalonde and Strazielle, 2007; Martin et al, 2010; Sacchetti et al, 2005; Thach et al, 1992).

*Correspondence: Professor BKC Chow, School of Biological Sciences, University of Hong Kong, 4NOI, Kadoorie Biological Sciences Building, University of Hong Kong, Pokfulam, Hong Kong SAR, China, Tel: +852 22990850, Fax: +852 25599||4, E-mail: bkcc@hku.hk

Received 15 April 2013; revised 16 December 2013; accepted 16 December 2013; accepted article preview online 19 December 2013
As the first discovered hormone (Bayliss and Starling, 1901), secretin (SCT) has been well studied with respect to its function in stimulating pancreatic secretion. In addition to the SCT immunoreactivity found in rat and pig brain extracts (O'Donohue et al, 1981), the expression of SCT and its receptor (SCTR) have also been detected in various brain regions, including the cortex, hippocampus, hypothalamus, and brainstem (Ng et al, 2002) across multiple developmental stages (Siu et al, 2005, 2006), leading to the hypothesis of a putative neuropeptide role for SCT. Our research group previously used SCT and SCTR knockout mouse models to demonstrate the endogenous release of SCT in the hypothalamus (Chu et al, 2006) and examine related central mechanisms in the regulation of water homeostasis (Chu et al, 2007; Chu et al, 2009; Lee et al, 2010) and food intake (Cheng et al, 2011). In behavioral studies, the central injection of SCT in rats led to decreased motor activity and lower novelobject approaches in an open field (Charlton et al, 1983). A recent study using SCTR knockout mice demonstrated hyperactivity and impaired rotarod performance (Nishijima et al, 2006). Other studies showed that stereotypic circular movements in Japanese waltzing mice were attenuated by central injection or intranasal application of SCT, which increased the horizontal movement and ambulation distance but did not influence the exploratory behavior (Heinzlmann et al, 2012; Koves et al, 2011). However, a comprehensive study focusing on fine motor control and motor learning functions of SCT has not been reported. 
Functioning as the integration center of the cerebellar cortex, Purkinje cells have a vital role in motor movement and coordination, spatial memory and learning, and even certain cognitive behavior (Sacchetti et al, 2005; Thach et al, 1992). Previous studies have demonstrated that SCT can be endogenously released from rat cerebellar Purkinje cells following $\mathrm{Ca}^{2+}$ entry and functions as a retrograde neuropeptide to potentiate GABAergic inhibitory postsynaptic currents (IPSCs) from basket cells via specific binding to SCTR (Lee et al, 2005; Yung et al, 2001). One possible mechanism of this potentiation was suggested to be the suppression of potassium channel Kv1.2 trafficking in both basket cell axon terminals and Purkinje dendrites after SCT application (Williams et al, 2012). Based on the current literature, we hypothesize that SCT can modulate motor behaviors through its expression in Purkinje neurons. We therefore developed a Purkinje cell-specific SCT gene knockout (Pur-Sct ${ }^{-/-}$) mouse model, and in conjunction with SCT knockout (Sct $\left.{ }^{-1-}\right)$ and SCTR knockout $\left(\mathrm{Sctr}^{-{ }^{-}}\right)$ mice, we investigated the relationships among SCT, Purkinje cells, and motor behaviors.

\section{MATERIALS AND METHODS}

\section{Generating Pur-Sct ${ }^{-/-}$Mice and Animal Care}

The breeding strategy employed the Cre-LoxP recombination technique to generate Pur-Sct ${ }^{-/-}$mice according to standard methods (Gaveriaux-Ruff and Kieffer, 2007). Three lines of mice were used: (1) a Sct-Loxp mouse line in which the SCT-coding region is flanked by 2 LoxP sites was generated by homologous recombination. This mouse line was generated in our laboratory (Lee et al, 2010). (2) The Pcp2-Cre transgenic mouse line was a generous gift from $\mathrm{Dr}$ JD Huang (Department of Biochemistry, University of Hong Kong). The Cre recombinase gene under the $P c p 2$ promoter is specifically expressed in Purkinje cells and retinal bipolar cells (Zhang et al, 2004). (3) Sct-Loxp mice were mated with Pcp2-Cre mice, producing offspring in which the SCTcoding region was deleted in Purkinje cells only. We also used $S c t^{-9-}$ and $S c t r^{-1-}$ mice, which have been described elsewhere (Chu et al, 2007; Lee et al, 2010).

To genotype these transgenic mice, genomic DNA from juvenile tail cuts was extracted using the KAPA DNA extraction kit (KAPA Bio-Systems, Wilmington, MA, USA) according to the manufacturer's instructions. Pur-Sct ${ }^{-/}$ mice were identified by dual PCR amplification using primers for the Pcp2-Cre sequence (Zhang et al, 2004) or the Sct-Loxp sequence (three primers: SCT-Llox5-F, 5'-ATAAG CGGCCGCGATTTGAGTTTCGGTGCTGG-3'; SCT-Rlox5-F, 5'-ATAAGCGGCCGCGAGTGCCACCTTGCCCTG-3'; and SCTRlox3-R, 5'-GTCGTCGACGGTTTGGGGAGCCAGTATCT-3').

All animal experimental protocols were pre-approved by the Committee on the Use of Living Animals in Teaching and Research of the University of Hong Kong. All mice were kept in a temperature-controlled room with a 12/12-h normal light/dark cycle (light 0700 to 1900 hours) and food and water provided ad libitum. Mice were housed in groups of four to six of the same sex per cage. All experiments used 2-month-old male mice (20-25 g) that had been backcrossed with $\mathrm{C} 57 \mathrm{BL} / 6 \mathrm{~J}$ mice for at least 10 generations $(N \geqslant 10)$, unless otherwise specified.

\section{Immunohistochemical (IHC) Staining and In Situ Hybridization}

IHC staining was performed as described elsewhere (Chu et al, 2007; Lee et al, 2010). Mice were euthanized, and whole brains were fixed in $3.7 \%$ paraformaldehyde, embedded in paraffin, and sectioned into $8 \mu \mathrm{m}$ parasagittal slices. After deparaffinization, non-specific binding was blocked with $10 \%$ normal goat serum followed by rabbit anti-SCT IgG (1:500 dilution; Bioss, Woburn, MA, USA) incubation overnight at $4{ }^{\circ} \mathrm{C}$. Signals were detected using the SuperPicTure Polymer Detection Kit (Invitrogen, Carlsbad, CA, USA) with horseradish peroxidase-conjugated antirabbit IgG and DAB chromogenic substrates. After counterstaining with hematoxylin (Vector Labs, Burlingame, CA, USA), images were captured using a Nikon $80 \mathrm{i}$ microscope. In situ hybridization using DIG-labeled SCT mRNA was performed as previously described (Cheng et al, 2011). In brief, anti-sense SCT DIG-labeled RNA was generated using a DIG RNA labeling kit (Roche, Basel, Switzerland) from a mouse Sct cDNA construct. Paraffin-based parasagittal brain sections were treated with $0.2 \mathrm{M} \mathrm{HCl}$ and $5 \mu \mathrm{M}$ Proteinase $\mathrm{K}$ followed by incubation with pre-hybridization buffer (10 mm Tris, $600 \mathrm{~mm} \mathrm{NaCl,} 1 \mathrm{~mm}$ EDTA, 0.25\% SDS, $10 \%$ dextran sulfate, $1 \times$ Denhardt's solution, and $200 \mathrm{mg} / \mathrm{ml}$ yeast tRNA in $50 \%$ formamide) and hybridization buffer containing $1 \mathrm{ng} / \mu \mathrm{l}$ DIG-labeled antisense Sct probes at $50{ }^{\circ} \mathrm{C}$ overnight. A negative control was performed using hybridization buffer containing $20 \times$ unlabeled antisense Sct probes. Signals were visualized with anti-DIG IgG and NBT/BCIP substrate (Roche, Swiss).

\section{Open Field Test}

As previously described (Karl et al, 2003), one mouse was put into a transparent plastic $25 \mathrm{~cm} \times 25 \mathrm{~cm}$ box and was allowed to freely explore the field in a single 60 -min session. Parameters were recorded and analyzed, including the velocity, time in movement, time in the central/marginal zone, and average distance toward the zone border or field center.

\section{Wire Hanging Test}

The wire hanging test was performed using previously reported procedures (Mocholi et al, 2011; Paylor et al, 1998). In brief, a metal wire mesh $(0.5 \mathrm{~mm}$ diameter, $5 \mathrm{~mm}$ wide grid) was fixed horizontally $30 \mathrm{~cm}$ above the table. One mouse was made to grab the wire mesh while upside-down. The latency until the animal fell down was recorded. A maximum time of $180 \mathrm{~s}$ was used, and unsuccessful animals were given a second chance. There were two sessions for each individual with a 30-min interval, and the longer time was used.

\section{Vertical Climbing Test}

According to previously described procedures (Brooks and Dunnett, 2009), a metal wire-mesh (0.5 mm diameter, $5 \mathrm{~mm}$ wide grid) was placed vertically $30 \mathrm{~cm}$ above the table. Each mouse started from the bottom of the mesh with its head facing downwards. After the mouse was released, the time 
required for it to climb all the way to the top was recorded. A maximum time of $60 \mathrm{~s}$ was applied for animals that could not successfully complete this task. Two sessions were performed for each mouse with a 30-min interval, and the shorter time was recorded.

\section{Rotarod Test}

The rotarod test was performed as previously described (Brooks and Dunnett, 2009; Karl et al, 2003). Our study used a wooden $\operatorname{rod}(3 \mathrm{~cm}$ diameter $\times 8 \mathrm{~cm}$ length $)$ with an adjustable speed. The test began with a short training session, in which the mouse was trained to stay on a still rod for at least $30 \mathrm{~s}$. Each animal was then enrolled into either of the following two paradigms: (1) constant speed test, in which the rod speed was fixed at 20 r.p.m.; (2) accelerating speed test, during which the rod was accelerated from 10 to 40 r.p.m. over $5 \mathrm{~min}$. The end point was defined as the time when any paw of the mouse left the rod. There were three or six daily test sessions for each mouse in the constant speed and accelerating speed paradigms, respectively.

\section{Postnatal Neurobehavioral Analysis}

Selected developmental markers were evaluated on each day beginning from postnatal day 4 (P4). A detailed description of each behavior is available in Chapter 8, Current Protocols of Neuroscience (Heyser et al, 2001). Two cerebellar neuron reflexes were tested: (1) righting reflex. The juvenile animal was put down on its back, and the positive reflex was identified as the ability to turn the body upright within $30 \mathrm{~s}$ (Mocholi et al, 2011; Yu et al, 2008). (2) Negative geotaxis reflex. The mouse was put on a $45^{\circ}$ inclined plane with its head facing downwards. Mice that could turn around and crawl up the slope were considered as having the positive reflex (Mocholi et al, 2011).

\section{Statistical Analysis}

All data are presented as the mean \pm SEM unless otherwise specified. For data having a normal distribution, a twotailed unpaired Student's $t$-test was used for betweensample comparisons and a one-way analysis of variance (ANOVA) test was used to identify any significant differences among multiple groups. When two independent factors were involved, two-way ANOVA was adopted to indicate effects from either factor. ANOVA test was followed by post-hoc test for between-two-group comparisons. For data with non-normal distribution, a two-sample K-S test was applied for between-group comparisons. All statistical analyses and the production of graphs were performed using SPSS (Statistical Package for the Social Sciences) v16.0 software (IBM, Armonk, NY, USA) and GraphPad Prism v5 software (La Jolla, CA, USA).

\section{RESULTS}

The SCT Gene Is Specifically Knocked Out in Purkinje Cells

We performed IHC staining and in situ hybridization studies on parasagittal brain slices from wild-type (WT) and Purkinje-SCT knockout (Pur-Sct ${ }^{-/-}$) mice to examine SCT gene transcripts and peptide expression in different neurons. Minimal levels of SCT transcript and peptide were found in cerebellar Purkinje cells of Pur-Sct $t^{-1-}$ mice (Figures 1c and e), in contrast to the strong expression present in WT cells (Figures $1 \mathrm{~b}$ and d). SCT was present in the soma and dendrites of Purkinje cells in WT mice (Figure 1b, enlarged views). There was prominent SCT expression in the deep cerebellar nuclei, which are downstream neurons of Purkinje cells, as well as in the hippocampus and hypothalamus from both WT and Pur-Sct ${ }^{-/}$(Figures 1f-i). These findings substantiate the specificity of SCT gene knockout only in Purkinje cells of Pur-Sct ${ }^{-/-}$mice.

\section{General Motor Functions and Normal Anxiety Levels Are Intact in SCT Knockout Mice}

An open field test was employed to observe spontaneous movements as an indicator of the motor function and stress level (Seo et al, 2010) before the performance of complex behavioral tasks. We analyzed the amount of time that the animal spent in central vs marginal regions (Figures 2a and $b$ ) and the average distance toward the zone center or border (Figures $2 \mathrm{c}$ and $\mathrm{d}$ ) and found similar results for all genotypes (one-way ANOVA, $p>0.05$ in all cases). No significant difference in velocity was also observed among WT, SCT-LoxP $\left(S c t^{f l f l}\right), S c t^{-1-}, S c t r^{-1-}$, and Pur-Sct ${ }^{-1-}$ mice (Figure 2e, one-way ANOVA, $p>0.05$ ), suggesting again that general motor functions were intact with the knockout of SCT or SCTR. In summary, the general motor ability and stress level were found intact in Purkinje cellspecific SCT knockouts.

\section{Neuromuscular Strength Is Weak in Pur-Sct ${ }^{-1-}$, $S c t^{-/-}$, and $S c t r^{-1-}$ Mice}

The wire hanging test was employed to measure the neuromuscular strength of transgenic mice. Both WT and $S c t^{f l / f l}$ animals had significantly higher holding latencies $\left(144.9 \pm 8.1\right.$ and $122.9 \pm 5.3 \mathrm{~s}$, respectively) than $S c t^{-/-}$, Sctr $^{-1-}$, and Pur-Sct ${ }^{-I-}$ mice $(34.1 \pm 9.4,52.0 \pm 15.8$, and $38.0 \pm 4.7 \mathrm{~s}$, respectively) by one-way ANOVA followed by post-hoc analysis (post-hoc Tukey's test, $p<0.001$ in all three cases). Comparisons among the three knockout groups showed no significant difference $(p>0.05)$, suggesting that the reason for these mice sharing the same phenotype of weak neuromuscular strength demonstrated in the wire hanging task (Miyakawa et al, 2001) is the disruption of the SCT-SCTR axis in Purkinje cells.

\section{SCT Deficiency Leads to Impaired Motor Coordination}

The vertical climbing test provides a simple assessment of impairments in motor and sensorimotor abilities (Brooks and Dunnett, 2009). Under this test paradigm, WT and $S c f^{f / f l}$ animals required $14.4 \pm 4.9$ and $19.3 \pm 4.5 \mathrm{~s}$, respectively, to climb up to the top of the vertical wire mesh. In contrast, $\mathrm{Sct}^{-1-}, \mathrm{Sctr}^{-1-}$, and Pur-Sct ${ }^{-1-}$ mice required $42.3 \pm 4.2$, $47.0 \pm 4.3$, and $50.8 \pm 4.9 \mathrm{~s}$, respectively, to complete this task (Figure 3b; post-hoc Tukey's test, $p<0.001$ in all knockout mice when comparing to wild types). In summary, the SCT gene knockout in Purkinje cells results in impaired motor coordination in mice. 
a

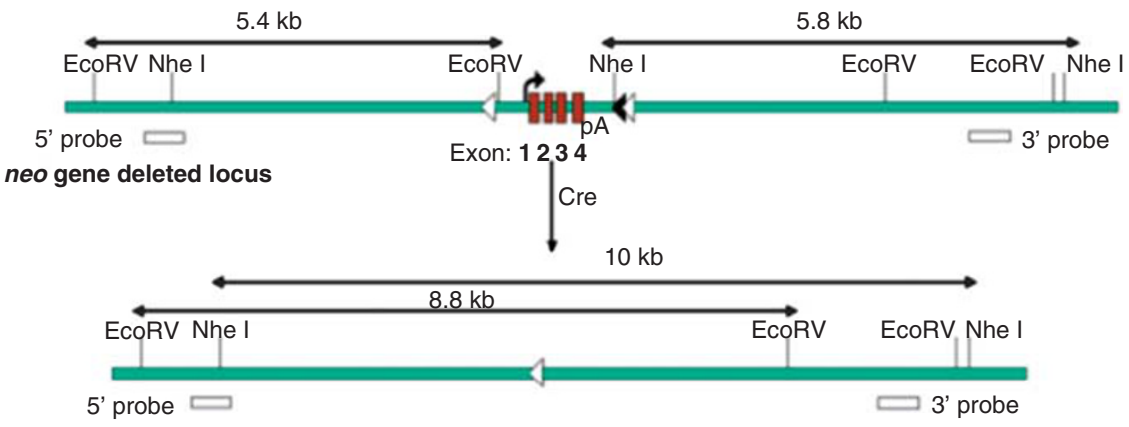

Sct gene KO
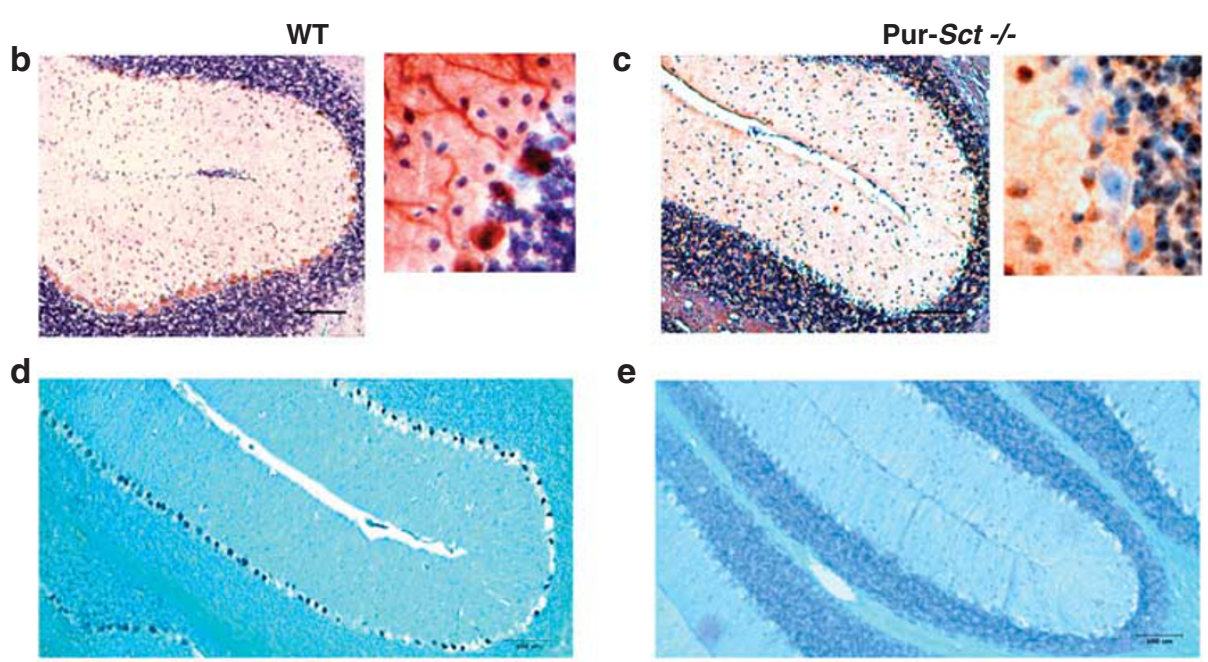

e
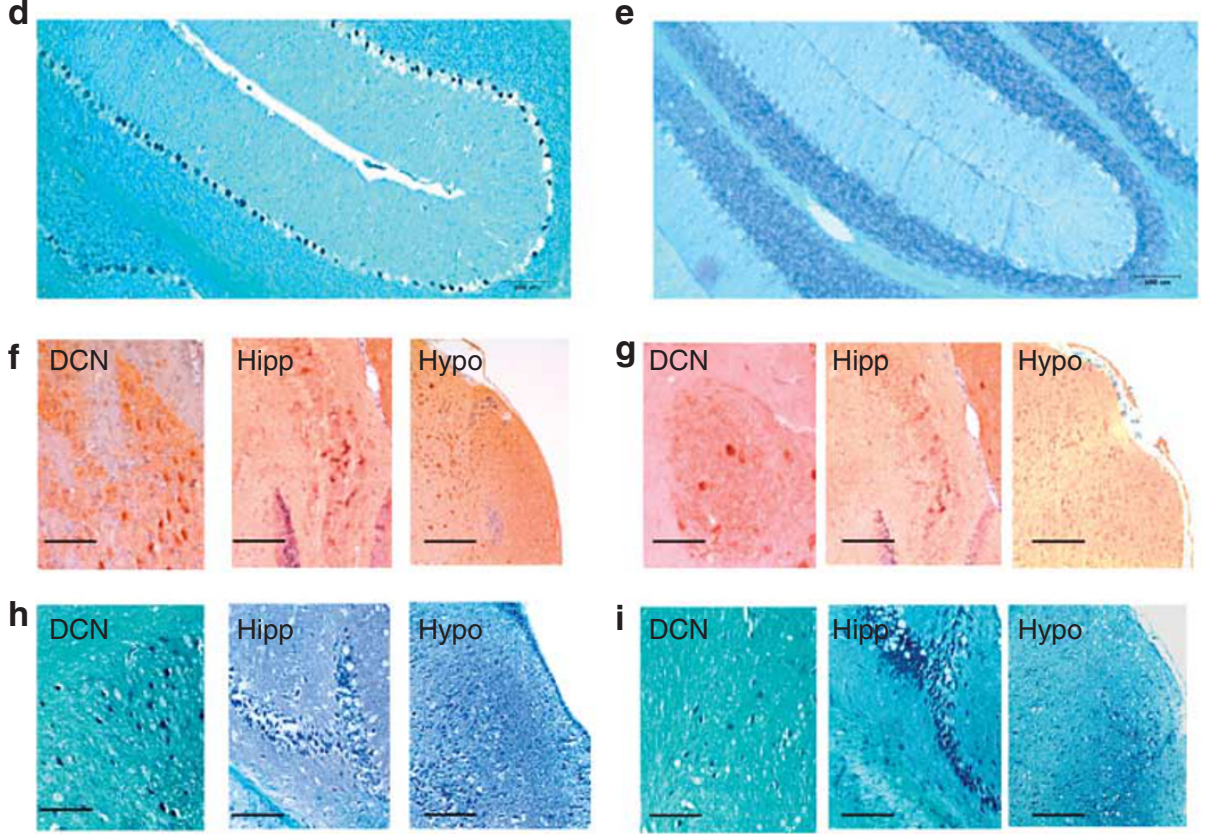

Figure I Purkinje cell-specific SCT knockout (Pur-Sct ${ }^{-{ }^{-}}$) by Cre recombination. (a) LoxP sequences flanking the SCT-coding region, along with an frtneo-frt cassette, were introduced into the mouse genome. The expression of Cre recombinase in Purkinje cells deletes the SCT gene. (b-e) Pur-Sct ${ }^{-1-}$ and WT cerebellum sagittal sections were stained using SCT antibody (b and c) and DIG-Sct mRNA probe (d, e). There was minimal expression of SCT transcript or peptide in Purkinje cells from Pur-Sct ${ }^{-1-}(c, e)$. In contrast, WT mice showed abundant secretin transcript and protein signals in Purkinje neurons (b and d). Both WT ( $f$ and $h$ ) and Pur-Sct ${ }^{-1-}(g, i)$ mice exhibited high levels of SCT transcript and protein expression in deep cerebellar nuclei (DCN), hippocampus (Hipp), and hypothalamus (Hypo), indicating the specificity of the SCT knockout in Purkinje cells. GL, granular cell layer; P, Purkinje cells. Scale bar in (b-i), $100 \mu \mathrm{m}$.

\section{Motor Learning Functions Are Impaired in Pur-Sct ${ }^{-/-}$ Mice}

To further investigate the motor learning functions of Pur$S c t^{-1-}$ mice, the rotarod test was performed using a constant speed task and an accelerating speed task. In both scenarios, WT and Sct fl/fl animals showed longer latencies, staying on the rod starting from their first trials (constant speed: $49.0 \pm 8.5$ and $35.4 \pm 12.0 \mathrm{~s}$; accelerating speed: $116.6 \pm 10.2$ and $110.8 \pm 10.8 \mathrm{~s}$ ) when compared with $S c t^{-/-}$, $\mathrm{Sctr}^{-1-}$, and Pur-Sct ${ }^{-1-}$ mice (constant speed: $\mathrm{Sct}^{-{ }^{-}}$ $15.6 \pm 3.7, \mathrm{Sctr}^{-1-} 13.0 \pm 1.5$, and Pur-Sct ${ }^{-1-} 18.0 \pm 3.5 \mathrm{~s}$, respectively; accelerating speed: $S c t^{-1-} 79.3 \pm 15.7$, Sctr ${ }^{-1-}$ $45.1 \pm 17.0$, and Pur-Sct ${ }^{-1} 83.3 \pm 16.7 \mathrm{~s}$, respectively). Twoway ANOVA using genotype as the main factor revealed significant differences in rotarod performances between 

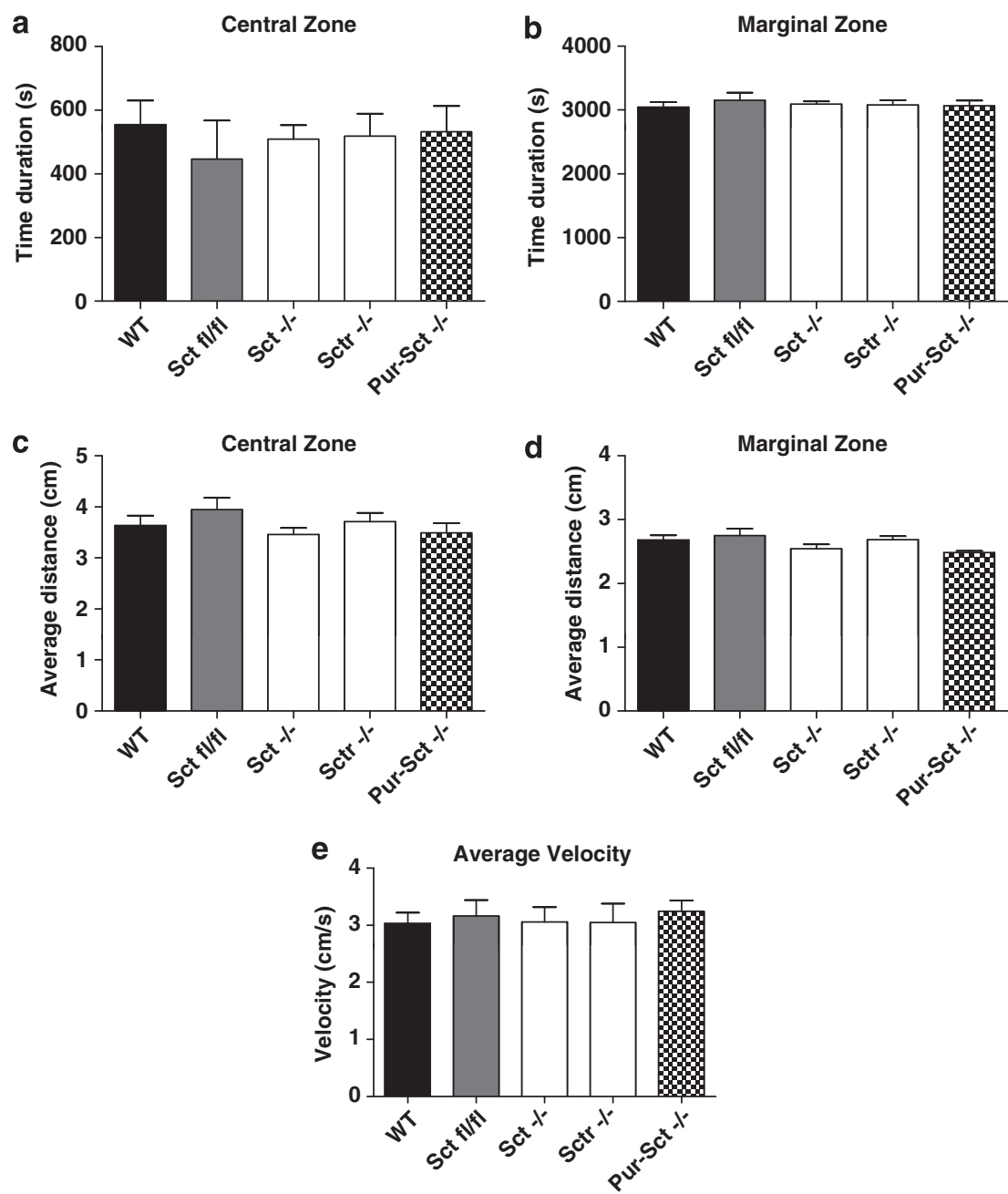

Figure 2 Open field test. Mice showed a similar duration in the center (a) or marginal regions (b) and similar average distances toward the zone center (c) or zone border (d) among all assessed genotypes. In addition, the average velocity (e) was constant across all genotypes. $N=9-10$ per group; all values are expressed as the mean \pm SEM.

knockout and wild types $(p<0.001$ in both cases). With repeated trials, the divergence between gene knockout groups and controls became even more significant (Figures $3 \mathrm{c}$ and $\mathrm{d}$ ): using latencies from the first trial as the base level, we found minor increases of latency in knockout mice at the last trial, whereas the control animals showed more robust improvements (constant speed: WT $39.4 \pm 20.2 ;$ Sct $^{\text {flfl }} 58.8 \pm$ 14.4; Sct ${ }^{-/-}$8.2 \pm 4.9; Sctr ${ }^{-/-} 11.6 \pm 5.8 ;$ Pur-Sct $^{-/-} 1.0 \pm$ $1.5 \mathrm{~s}$; accelerating speed: WT 137.1 $\pm 24.0 ; S c t^{f l f l} 107.0 \pm 27.9$; Sct ${ }^{-1-} 54.6 \pm 24.2 ;$ Sctr $^{-/-} 30.0 \pm 6.0 ;$ Pur-Sct $^{-/-} 11.0 \pm$ $10.1 \mathrm{~s})$. Two-way ANOVA of the data is consistent with the post-hoc test, showing differential improvements of these genotypes with respect to the number of trial sessions $(p<0.01)$. This trend demonstrates the compromised motor learning abilities in Sct ${ }^{-/-}, \mathrm{Sctr}^{-/-}$(post-hoc Bonferroni comparison, $p<0.01)$, and, most significantly, in Pur-Sct ${ }^{-1-}$ $(p<0.001)$ when comparing to wild types.

\section{Pur-Sct ${ }^{-1-}$ Juveniles may have Delayed Cerebellar Development}

After initial observation of impairments in motor coordination and learning in Pur-Sct ${ }^{-/-}$, we further investigated whether such deficits occurred in juveniles. A set of developmental milestones was employed to screen Pur$S c t^{-1}$ and $S c t^{f l f l}$ littermates after birth. Among all these markers, Pur-Sct ${ }^{-1-}$ juveniles had a later onset of surface righting and negative geotaxis reflexes (Figure 4a). This difference was replicated using a second cohort of mice (Figures $4 \mathrm{~b}$ and c; medium $=\mathrm{P} 7$ vs $\mathrm{P} 6$ in righting and $\mathrm{P} 9 v s$ P7 in negative geotaxis; $p<0.001$ using non-parametric K-S test). Both reflexes have previously been used to evaluate cerebellar development (Mocholi et al, 2011; Yu et al, 2008). Our data therefore suggest that SCT knockout in Purkinje cells can lead to neural developmental delays in the cerebellum.

\section{Pur-Sct ${ }^{-/-}$Mice Show no Significant Signs of Neural Degeneration}

We further sought to determine whether SCT knockout leads to neural degeneration, which can be displayed as a deterioration of motor tasks in aging. An age-dependent study was thus employed on the same cohort of Pur-Sct ${ }^{-1-}$ and $S c t^{f l f l}$ littermates to measure their performances in wire hanging and accelerating rotarod tests. Analysis of the data 

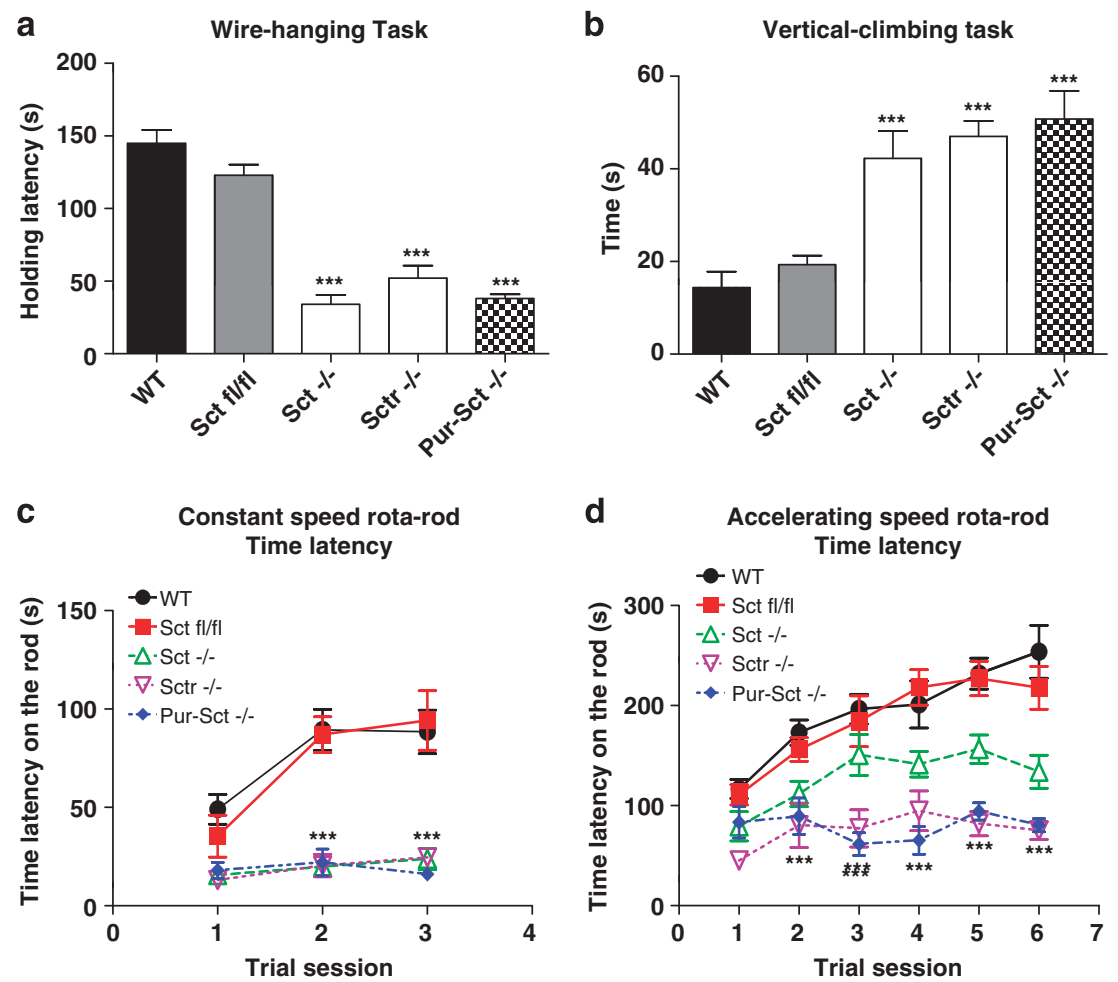

Figure 3 Impaired fine motor control abilities in mice lacking secretin. Four behavior tasks were performed: (a) wire-hanging latency; (b) time to climb a vertical wire mesh; (c) time on a constant speed rotarod; (d) time on an accelerating rotarod. (a-d) Comparisons between Pur-Sct ${ }^{-1-}$, Sct $t^{f / f f}$, and WT as well as Sct ${ }^{-1-}$ and Sctr ${ }^{-1-}$ mice. Pur-Sct ${ }^{-1-}$ exhibited significantly lower neuromuscular strength (a), fine motor control (b), balance functions, and motor learning (c, d). All of these abnormalities also occurred in Sct ${ }^{-1-}$ and Sctr ${ }^{-1-}(a-d)$. Similar performances were found in Sctl/f and WT (a-d), indicating

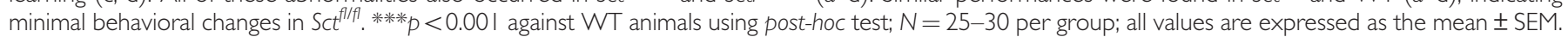

found: (1) significant shorter latencies were observed in Pur-Sct ${ }^{-1}$ compared with $S c t^{f l / f l}$ littermates at all age groups (Figures $4 \mathrm{~d}$ and e; two-way ANOVA using genotype as the main factor, $p<0.001$; post-hoc Bonferroni comparison, $p<0.05)$; (2) age-dependent motor deficits occurred in both Pur-Sct ${ }^{-1-}$ and Sct $t^{f l f l}$ littermates (two-way ANOVA using age as the main factor, $p<0.01$ ); and (3) no difference in terms of age-dependent motor impairments existed between Pur-Sct ${ }^{-1-}$ and Sct $t^{f l f l}$ (two-way ANOVA for interaction effect between age and genotype factors, $p>0.05$ ). Taken together, age-dependent motor deficits existed within each genotype but not between two genotypes. However, wire hanging test showed decreased latencies starting from month 2 until month 4 in Pur-Sct ${ }^{-1-}$ but not in $S c f^{f l / f l}$, which began to display significant motor deficits only after month 6 (Figure 4d). Therefore, our data indicate that SCT knockout in Purkinje neurons could lead to early onset motor neuron degeneration.

\section{DISCUSSION}

Using the Cre-LoxP recombination technique, we successfully generated a Purkinje cell-specific SCT knockout mouse model. This is the first time that SCT expression has been manipulated in specific cells and hence provides a more powerful model to investigate the exact function of SCT in specific cells. In WT mice, SCT was strongly expressed in the soma and dendrites of Purkinje cells (Figures $1 \mathrm{~b}$ and $\mathrm{d}$ ), consistent with results obtained from a previous rat cerebellum study (Yung et al, 2001). In Pur-Sct ${ }^{-/-}$mice, SCT was still expressed in the deep cerebellar nuclei, hippocampus, and hypothalamus (Figures $1 \mathrm{f}-\mathrm{i}$ ) as previously described ( $\mathrm{Ng}$ et al, 2002), confirming the Purkinjespecific knockout of SCT. The wide distribution of SCT in the central nervous system indicates its multiple central functions, some of which have been demonstrated by our research group, including water homeostasis (Chu et al, 2009; Lee et al, 2010) and food intake (Cheng et al, 2011).

Our behavioral analysis showed that SCT knockout in Purkinje cells led to impairments in neuromuscular strength, motor coordination, and motor learning functions. Such motor dysfunctions were unlikely to have been caused by impairments in general motor functions or increases in anxiety levels because the mice showed unchanged movement velocity and a propensity toward the zone border in the open field (Figure 2). We studied motor coordination and learning functions using three different assays. First, $S c t^{-1-}, S_{c t r}{ }^{-1-}$, and Pur-Sct ${ }^{-1-}$ mice spent significantly shorter times holding the metal wire (Figure $3 \mathrm{a}$ ), suggesting impaired neuromuscular strength (Miyakawa et al, 2001). Subsequently, the vertical climbing test provided evidence for impairments in fine motor coordination (Brooks and Dunnett, 2009) because of SCT knockout, as a majority of the knockout mice climbed slowly, stayed still, or even fell off the wire. Profound impairments in vertical climbing 

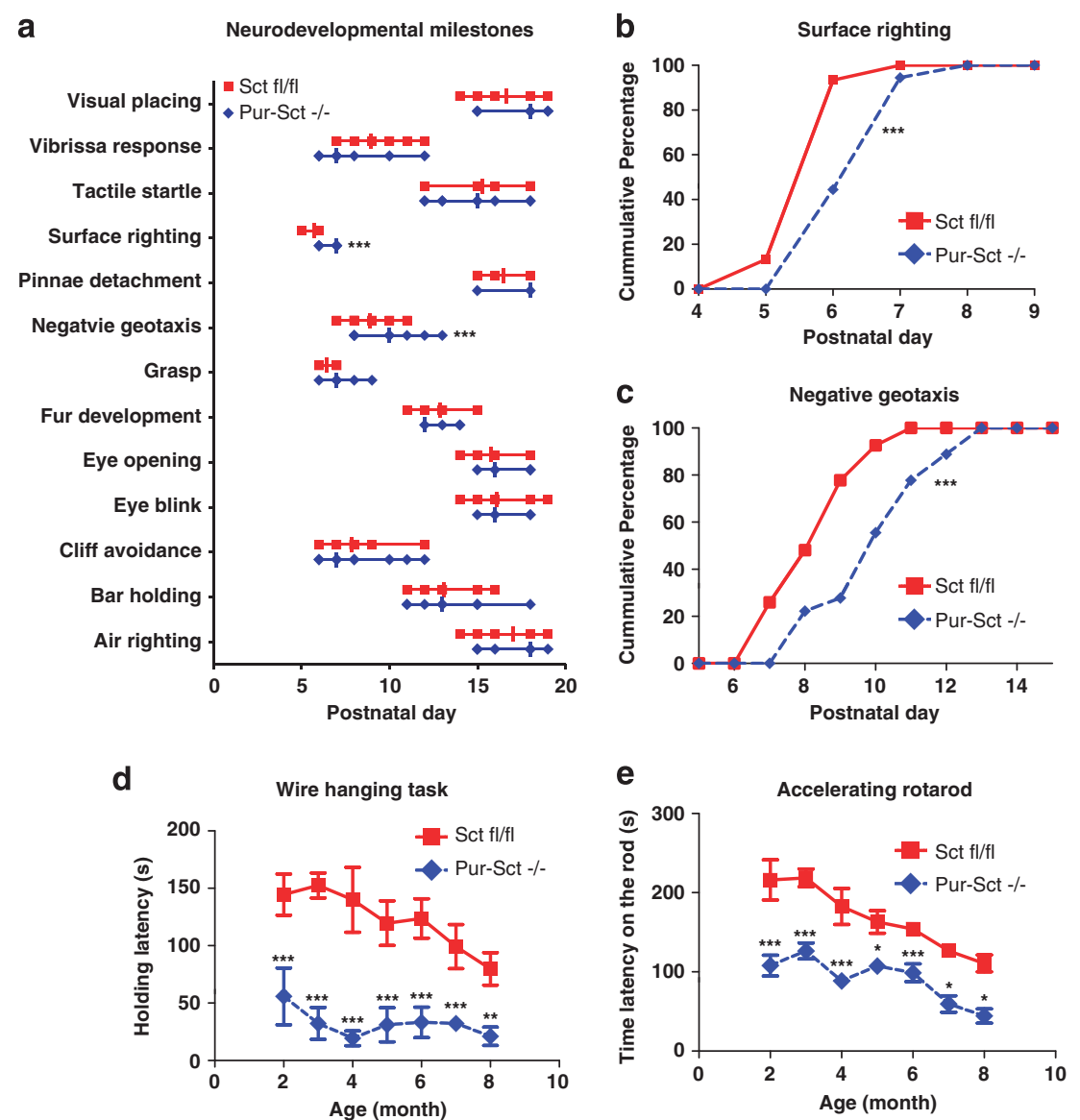

Figure 4 Age-dependent effects of Purkinje-specific secretin knockout. (a) A full battery of developmental milestones for juvenile mice was checked after birth. Data are showed in aligned dot plot with medians \pm ranges in postnatal days. Accumulated frequencies of juveniles with positive surface righting (b) and negative geotaxis (c) reflexes were plotted against postnatal days. Age-dependent wire hanging (d) and accelerating rotarod (e) performance were

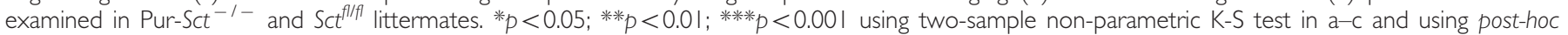
Bonferroni test followed two-way ANOVA in $\mathrm{d}$ and e. $\mathrm{N}=15-20$ per group. All values in $\mathrm{d}$ and e are expressed as the mean \pm SEM.

have been reported in mice with reduced neurite formation (Mocholi et al, 2011) and in knock-in Huntington's disease transgenic mice (Hickey et al, 2008). Moreover, rotarod motor latencies for $S c t^{-1-}, S_{c t r}{ }^{-1-}$, and Pur-Sct ${ }^{-/-}$mice increased slightly after repeated trials (Figures $3 c$ and $d$ ), illustrating motor learning deficits. The abnormal behavioral phenotypes that we discovered, combined with results from previous studies on the SCT-mediated potentiation of rat Purkinje cell IPSCs (Yung et al, 2001) and the specific knockout of SCT in Purkinje cells of the mouse model, strongly support the involvement of the SCT-SCTR axis in motor coordination and learning abilities through the modulation of Purkinje cell neural transmission.

There have been few previous studies discussing SCT and animal behaviors. In contrast to our open field assay, in which SCT knockout had no effects on horizontal activity, an intracerebroventricular (ICV) injection of SCT has been shown to induce hypoactivity in rats (Charlton et al, 1983). This result, however, can be explained by the higher anxiety level, which led to a decreased propensity for rats to initiate movements (Charlton et al, 1983) and thus has little relevance to motor functions. In another study, SCTR knockout mice had motor-learning deficits in rotarod and water-maze tests, which can largely be explained by the impaired synaptic plasticity and reduced dendritic spines in CA1 of the hippocampus (Nishijima et al, 2006). Our Pur$\mathrm{Sct}^{-{ }^{-}}$mice had similar learning deficits, suggesting a role for cerebellar SCT in motor learning. Moreover, ICV injection or intranasal application of SCT attenuated repetitive circular movements and improved horizontal activities in Japanese waltzing mice (Heinzlmann et al, 2012; Koves et al, 2011). This study illustrated the role of SCT in restoring normal behavioral patterns, which is also supported by our studies in which SCT knockout led to impaired motor regulation. One recent study reported enhancement of eye blink conditioning in rats after ICV SCT injection (Williams et al, 2012), suggesting the role of SCT in motor learning. Taken together, past studies provided correlations between SCT and rodent behaviors but lacked a comprehensive description of motor functions. The present report provides the first direct illustration of the impairments of motor coordination and motor-learning functions upon SCT knockout in Purkinje neurons.

The time frame of such motor disabilities is important to understand the effect of SCT on cerebellar neurodevelopment. In Pur-Sct ${ }^{-\prime-}$ mice, the Cre recombinase began to 
show activity at P6 and peaked at P15 (Zhang et al, 2004). Our behavioral tests using 2-month-old animals therefore reflected the consequences of complete elimination of SCT in Purkinje cells. To assess the early-stage effects of the SCT knockout, righting and negative geotaxis reflexes were assessed in Pur-Sct ${ }^{-1-}$ juveniles. The delayed onset of both motor reflexes (Figures $4 \mathrm{a}-\mathrm{c}$ ) compared with $S c t^{\text {fl/fl }}$ littermates illustrated the effects of SCT in neurobehavioral development (Mocholi et al, 2011; Yu et al, 2008). The time window for the development of these two reflexes (approximately P6-P9) coincides with the onset of Cre activity (Zhang et al, 2004) and is an important stage for Purkinje cell development (Erickson et al, 2007). Anatomical studies provided preliminary information regarding the role of SCT in cerebellar development; eg, SCT was shown to stimulate neurite outgrowth in vitro (Kim et al, 2006) and to protect cerebellar granular cell progenitors from ethanol toxicity in mice (Hwang et al, 2009). All of these studies, consistent with our results, support the hypothesis that SCT gene deletion may alter the pattern of cerebellar development, leading to persistent functional deficits. We also investigated differences in motor coordination and learning abilities between Pur-Sct ${ }^{-1-}$ and $S c t^{f l f l}$ littermates in various age groups. The results showed consistent motor deficits between control and knockout mice in each compared age group (Figures $4 \mathrm{~d}$ and e). It is interesting that in wire hanging task, significant performance decrease occurred earlier in Pur-Sct ${ }^{-/-}$than that in $S c t^{f l f l}$ littermates. Therefore, knockout of SCT in Purkinje neurons possibly leads to early onset of neural degeneration. This hypothesis could be partially supported by the protective role of SCT against ethanol toxicity as aforementioned (Hwang et al, 2009). However, no direct evidence regarding neural protection function of SCT in mature brains has been implicated, and further anatomical and pathological observations are required to substantiate this hypothesis.

The cellular mechanism underlying the motor behavioral abnormalities remains unclear. Potential effects on two pathways, ie, neural transmission and/or neural development, may help to explain the motor coordination and learning deficits. For neural transmission, we have demonstrated that SCT can potentiate GABAergic IPSCs in basketPurkinje cell synaptic transmission from rats (Yung et al, 2001). The underlying mechanism has been suggested to be the suppression of potassium channel Kv1.2 trafficking in basket cell-Purkinje neuron synapses (Williams et al, 2012). As Kv1.2 is expressed in both basket cell axon terminal and Purkinje dendrites, this finding raised the involvement of both presynaptic GABA release resulting from reduced Kv1.2 ion currents (Southan and Robertson, 1998) and postsynaptic facilitation of parallel fiber-Purkinje neuron long-term depression, which is widely known as foundations of motor learning (Lamont and Weber, 2012). Our initial hypothesis about SCT's role in motor behaviors can be further strengthened by observations in transgenic mice with Purkinje neuron deficits. For example, GABA transporter subtype-1 knockout mice showed a slower decay of spontaneous IPSCs and concurrent poor performance in the rotarod test (Chiu et al, 2005). Poor wire hanging performance was also reported in Nagoya mice with malfunctioning Cav2.1 channels, which are critical in parallel fiber-Purkinje cell synaptic transmission (Takahashi et al, 2009). In addition, we found SCT expression in the deep cerebellar nuclei (Figures $1 \mathrm{f}-\mathrm{i}$ ), which receive inhibitory outputs from Purkinje cells. Therefore, SCT may modulate both of basket-Purkinje and Purkinje-deep cerebellar nuclei synaptic transmission via pre- and post-synaptic potentiation mechanisms. In the neural developmental scenario, SCT can function as a neurotrophic factor both in vitro (Kim et al, 2006) and in vivo for cerebellar granular cells (Hwang et al, 2009) and hippocampus neural progenitors (Jukkola et al, 2011). It is worth noting that RhoE-deficient mice with compromised neurite formation had profound impairments in wire hanging, vertical climbing, righting reflex, and negative geotaxis reflex (Mocholi et al, 2011), all of which were displayed in our Pur-Sct ${ }^{-1-}$ juveniles. Therefore, SCT may function as a neurotrophin, contributing to the migration/differentiation of Purkinje, and other motor neurons in maintaining normal motor phenotypes. Taken together, these data indicate that SCT may act in neural transmission and/or neural developmental processes to help accomplish motor functions. Additional comprehensive studies including electrophysiological recording, morphological examination, and neuron cell activity quantification are required to illustrate the mechanism of SCT in regulating motor behavioral phenotypes.

In conclusion, SCT knockout in mouse Purkinje cells caused impairments in neuromuscular strength, fine motor coordination, and motor learning abilities. Such deficits developed early and persisted with aging, suggesting that the Purkinje neurons were altered during development. These data support a novel function for SCT as a neuromodulator in the cerebellar cortex and indicate its future use as a pharmacological target in cerebellar dysfunction, although further studies are required to elucidate the cellular mechanisms.

\section{FUNDING AND DISCLOSURE}

The authors declare no conflict of interest.

\section{ACKNOWLEDGEMENTS}

This work was supported by HK government RGC Grant GRF 764510, 765113, and CRF grant HKU6/CRF/11G to BKC Chow. We thank Ms Lisa Zhang for her kind help in the open field test and Mr Hans $\mathrm{Ng}$ in helping preparing ISH experiments.

\section{REFERENCES}

Bayliss WM, Starling EH (1901). The movements and innervation of the small intestine. J Physiol 26: 125-138.

Brooks SP, Dunnett SB (2009). Tests to assess motor phenotype in mice: a user's guide. Nat Rev Neurosci 10: 519-529.

Charlton CG, Miller RL, Crawley JN, Handelmann GE, O’Donohue TL (1983). Secretin modulation of behavioral and physiological functions in the rat. Peptides 4: 739-742.

Cheng CY, Chu JY, Chow BK (2011). Central and peripheral administration of secretin inhibits food intake in mice through the activation of the melanocortin system. Neuropsychopharmacology 36: 459-471.

Chiu CS, Brickley S, Jensen K, Southwell A, McKinney S, CullCandy $S$ et al (2005). GABA transporter deficiency causes 
tremor, ataxia, nervousness, and increased GABA-induced tonic conductance in cerebellum. J Neurosci 25: 3234-3245.

Chu JY, Chung SC, Lam AK, Tam S, Chung SK, Chow BK (2007). Phenotypes developed in secretin receptor-null mice indicated a role for secretin in regulating renal water reabsorption. Mol Cell Biol 27: 2499-2511.

Chu JY, Lee LT, Lai CH, Vaudry H, Chan YS, Yung WH et al (2009). Secretin as a neurohypophysial factor regulating body water homeostasis. Proc Natl Acad Sci USA 106: 15961-15966.

Chu JY, Yung WH, Chow BK (2006). Endogenous release of secretin from the hypothalamus. Ann N Y Acad Sci 1070: 196-200.

Dow RS, Moruzzi G (1958). The Physiology and Pathology of the Cerebellum. University of Minnesota Press.

Erickson MA, Haburcak M, Smukler L, Dunlap K (2007). Altered functional expression of Purkinje cell calcium channels precedes motor dysfunction in tottering mice. Neuroscience 150: $547-555$.

Gaveriaux-Ruff C, Kieffer BL (2007). Conditional gene targeting in the mouse nervous system: Insights into brain function and diseases. Pharmacol Ther 113: 619-634.

Grusser-Cornehls U, Baurle J (2001). Mutant mice as a model for cerebellar ataxia. Prog Neurobiol 63: 489-540.

Heinzlmann A, Kiss G, Toth ZE, Dochnal R, Pal A, Sipos I et al (2012). Intranasal application of secretin, similarly to intracerebroventricular administration, influences the motor behavior of mice probably through specific receptors. J Mol Neurosci 48: 558-564.

Heyser CJ (2001). Assessment of developmental milestones in rodents. Curr Protoc Neurosci. John Wiley \& Sons, Inc.

Hickey MA, Kosmalska A, Enayati J, Cohen R, Zeitlin S, Levine MS et al (2008). Extensive early motor and non-motor behavioral deficits are followed by striatal neuronal loss in knock-in Huntington's disease mice. Neuroscience 157: 280-295.

Hwang DW, Givens B, Nishijima I (2009). Ethanol-induced developmental neurodegeneration in secretin receptor-deficient mice. Neuroreport 20: 698-701.

Ito M (1984). The modifiable neuronal network of the cerebellum. Jpn J Physiol 34: 781-792.

Ito M (2002). Historical review of the significance of the cerebellum and the role of Purkinje cells in motor learning. Ann N Y Acad Sci 978: 273-288.

Jukkola PI, Rogers JT, Kaspar BK, Weeber EJ, Nishijima I (2011). Secretin deficiency causes impairment in survival of neural progenitor cells in mice. Hum Mol Genet 20: 1000-1007.

Karl T, Pabst R, von Horsten S (2003). Behavioral phenotyping of mice in pharmacological and toxicological research. Exp Toxicol Pathol 55: 69-83.

Kim HS, Yumkham S, Kim SH, Yea K, Shin YC, Ryu SH et al (2006). Secretin induces neurite outgrowth of PC12 through cAMP-mitogen-activated protein kinase pathway. Exp Mol Med 38: 85-93.

Koves K, Kiss G, Heinzlmann A, Dochnal R, Manczinger M, Pal A et al (2011). Secretin attenuates the hereditary repetitive hyperactive movements in a mouse model. J Mol Neurosci 43: 109-114.

Lalonde R, Strazielle C (2007). Spontaneous and induced mouse mutations with cerebellar dysfunctions: behavior and neurochemistry. Brain Res 1140: 51-74.

Lamont MG, Weber JT (2012). The role of calcium in synaptic plasticity and motor learning in the cerebellar cortex. Neurosci Biobehav Rev 36: 1153-1162.

Lee SM, Chen L, Chow BK, Yung WH (2005). Endogenous release and multiple actions of secretin in the rat cerebellum. Neuroscience 134: 377-386.
Lee VH, Lee LT, Chu JY, Lam IP, Siu FK, Vaudry H et al (2010). An indispensable role of secretin in mediating the osmoregulatory functions of angiotensin II. FASEB J 24: 5024-5032.

Martin LA, Goldowitz D, Mittleman G (2010). Repetitive behavior and increased activity in mice with Purkinje cell loss: a model for understanding the role of cerebellar pathology in autism. Eur J Neurosci 31: 544-555.

Miyakawa T, Yared E, Pak JH, Huang FL, Huang KP, Crawley JN (2001). Neurogranin null mutant mice display performance deficits on spatial learning tasks with anxiety related components. Hippocampus 11: 763-775.

Mocholi E, Ballester-Lurbe B, Arque G, Poch E, Peris B, Guerri C et al (2011). RhoE deficiency produces postnatal lethality, profound motor deficits and neurodevelopmental delay in mice. PLoS one 6: e19236.

Ng SS, Yung WH, Chow BK (2002). Secretin as a neuropeptide. Mol Neurobiol 26: 97-107.

Nishijima I, Yamagata T, Spencer CM, Weeber EJ, Alekseyenko O, Sweatt JD et al (2006). Secretin receptor-deficient mice exhibit impaired synaptic plasticity and social behavior. Hum Mol Genet 15: 3241-3250.

O'Donohue TL, Charlton CG, Miller RL, Boden G, Jacobowitz DM (1981). Identification, characterization, and distribution of secretin immunoreactivity in rat and pig brain. Proc Natl Acad Sci USA 78: 5221-5224.

Paylor R, Nguyen M, Crawley JN, Patrick J, Beaudet A, Orr-Urtreger A (1998). Alpha7 nicotinic receptor subunits are not necessary for hippocampal-dependent learning or sensorimotor gating: a behavioral characterization of Acra7-deficient mice. Learn Mem 5: 302-316.

Sacchetti B, Scelfo B, Strata P (2005). The cerebellum: synaptic changes and fear conditioning. Neuroscientist 11: 217-227.

Seo HJ, Ham HD, Jin HY, Lee WH, Hwang HS, Park SA et al (2010). Chronic administration of monosodium glutamate under chronic variable stress impaired hypothalamic-pituitary-adrenal axis function in rats. Korean J Physiol Pharmacol 14: 213-221.

Siu FK, Sham MH, Chow BK (2005). Secretin, a known gastrointestinal peptide, is widely expressed during mouse embryonic development. Gene Expr Patterns 5: 445-451.

Siu FK, Sham MH, Chow BK (2006). The prenatal expression of secretin receptor. Ann N Y Acad Sci 1070: 561-565.

Southan AP, Robertson B (1998). Modulation of inhibitory postsynaptic currents (IPSCs) in mouse cerebellar Purkinje and basket cells by snake and scorpion toxin $\mathrm{K}+$ channel blockers. Br J Pharmacol 125: 1375-1381.

Swinny JD, van der Want JJ, Gramsbergen A (2005). Cerebellar development and plasticity: perspectives for motor coordination strategies, for motor skills, and for therapy. Neural Plast 12: 153-160.

Takahashi E, Niimi K, Itakura C (2009). Motor coordination impairment in aged heterozygous rolling Nagoya, Cav2.1 mutant mice. Brain Res 1279: 50-57.

Thach WT, Goodkin HP, Keating JG (1992). The cerebellum and the adaptive coordination of movement. Annu Rev Neurosci 15: 403-442.

Williams MR, Fuchs JR, Green JT, Morielli AD (2012). Cellular mechanisms and behavioral consequences of Kv1.2 regulation in the rat cerebellum. J Neurosci 32: 9228-9237.

Yu F, Liao Y, Jin Y, Zhao Y, Ren Y, Lu C et al (2008). Effects of in utero meso-2,3-dimercaptosuccinic acid with calcium and ascorbic acid on lead-induced fetal development. Arch Toxicol 82: 453-459.

Yung WH, Leung PS, Ng SS, Zhang J, Chan SC, Chow BK (2001). Secretin facilitates GABA transmission in the cerebellum. J Neurosci 21: 7063-7068.

Zhang XM, Ng AH, Tanner JA, Wu WT, Copeland NG, Jenkins NA et al (2004). Highly restricted expression of Cre recombinase in cerebellar Purkinje cells. Genesis 40: 45-51. 